\title{
Formación de habilidades en estudiantes de Derecho, una tarea de formar no de informar
}

\author{
Formation of abilities in law students, a task to form not to informing
}

\author{
Jorge Rondón Valdés ${ }^{1, *}$, Alcides Antúnez Sánchez ${ }^{1}$ \\ ${ }^{1}$ Universidad de Oriente, Cuba
}

\section{RESUMEN}

El artículo realiza un esbozo de los referentes teóricos que se han tenido en cuenta para el desarrollo de las habilidades en el proceso docente educativo, las principales ideas desde un enfoque histórico-cultural, se fundamenta en la necesidad e importancia de la formación de los estudiantes de Derecho para crear en estos habilidades sociales que le permitan demostrar sus competencias en el mercado laboral. Es realizada para ello una revisión de la introducción de la actividad científica dentro del proceso de enseñanza-aprendizaje que le permita el desarrollo de habilidades en la Universidad Cubana. A partir de la perspectiva de las nuevas tecnologías digitales en el desarrollo cognitivo de los jóvenes en sus valores, estilos de vida y expectativas educativas, para apropiarse de conocimientos 0 saberes jurídicos, y dotarlos de una serie de competencias y habilidades básicas que lo capaciten para el ejercicio de su profesión una vez que este acceda al mercado laboral desde los modos de actuación como fiscal, juez, abogado, asesor, profesor, entre otros. Para ellos nos auxiliamos en los métodos de la investigación como: el histórico lógico, el de análisis y síntesis, y el de inducción deducción.

Palabras clave: Competencias laborales, habilidades, proceso docente.

\begin{abstract}
The article makes an outline of the theoretical referents that have been taken into account for the development of skills in the educational process, the main ideas from a historical- cultural approach, is based on the need and importance of the training of students law to create in these social skills that allow you to demostrate your skills in the labor market. For this purpose a review of the introduction of scientific activity within the teaching learning process that allows the development of skills in the Universidad Cubana. From the perspective of the new digital technologies in the cognitive development of young people in their values, lifestyles and educational expectations, to appropriate knowledge or legal knoeledge, and provide them with a series of skills and basic skills that enable them to the exercise of his profession once he enters the labor market from the modes os action as prosecutor, judge, lawyer, counselor, professor, among, others. For them we help in research methods such as: the logical historical, the analysis and synthesis, and the induction deduction.
\end{abstract}

Keywords: Labor competitions, abilities, teaching process.

Historial del artículo:

Recibido, 04 de marzo 2016; aceptado, 23 de mayo de 2016; disponible en línea, 15 de julio de 2016

* Licenciado en Derecho. Máster en Derecho Civil. Profesor Asistente. Facultad de Ciencias Económicas y Sociales. Universidad de Granma. Doctorante de la Facultad de Derecho Universidad de Oriente, Cuba.

Correo: jrondonv@udg.co.cu 


\section{INTRODUCCIÓN}

La tarea de formar e informar a los juristas de estos tiempos, debe ser una preocupación de todos, se trata de formar un profesional que esté vinculado a la problemática social, para comprender la naturaleza real del Derecho como ciencia, valorado como un fenómeno en sí mismo, donde se logre combinar propósitos prácticos y profesionales con otros estrictamente teóricos o científicos.

El sistema de conocimientos, en la enseñanza del Derecho, no tiene sentido sin la existencia de los mecanismos de su estructuración, conservación y aplicación; por eso en el desarrollo del pensamiento aparecen las habilidades como expresión de la sistematización de procedimientos, técnicas y métodos.

Son estructuras del pensamiento, lógicas, motoras, que permiten asimilar, conservar, utilizar y exponer los conocimientos. Se estructuran en operaciones que constituyen su estructura técnica. Se forman y desarrollan a través de la ejercitación de las acciones y se convierten en modos de actuación que dan solución a tareas teóricas y prácticas (R. Álvarez, 1997).

También el modo de interacción del sujeto con los objetos, se dan en la actividad y la comunicación, constituyen el contenido de las acciones que el sujeto realiza con un objetivo, juegan un papel importante en la autorregulación del sujeto y en el conocimiento de sí mismo (Fuentes, 1999).

Debe destacarse que el proceso sistematización del contenido por los estudiantes está, a su vez, íntimamente ligada al desarrollo del pensamiento que se va produciendo en el proceso de enseñanza - aprendizaje; donde ese genera un vínculo entre el desarrollo intelectual y el proceso de asimilación.

La asimilación de cada nuevo procedimiento, requieren la formación de habilidades, lo que influirá en el desarrollo del pensamiento del estudiante. Todo esto motiva, delimitar habilidades que deben formarse en estudiantes de la carrera de Derecho, partiendo de su análisis teórico como categoría del proceso docente.

\section{¿Cómo definir las habilidades?}

Hay autores que plantean que para hablar de la naturaleza y clasificación de los contenidos del proceso de enseñanza aprendizaje, definen a la habilidad como "Uno de los tipos de contenido, junto con los conocimientos y las aptitudes" (De Zayas, 1997).

Otros la informan como el "Sistema de acciones y operaciones dominadas por el hombre, estructuradas en operaciones ordenadas y orientadas a la consecución de un objetivo, que le permiten interactuar con objetos determinados de la realidad y con otros sujetos. La habilidad es el modo de interacción del sujeto con el objeto, es el contenido de las acciones que el sujeto realiza, integradas por un conjunto de operaciones, que tiene un objetivo y que se asimila en el proceso" (Fuentes, 1998).

Ahora bien, las habilidades, valoran los autores, se adquieren en un proceso de apropiación de la estructura del objeto sobre el que recae la acción, de modo que en semejante proceso se desarrollan habilidades en el acto de aprender conocimientos y se aprenden conocimientos en el acto de desarrollar habilidades.

En la definición anterior queda delimitado que en la habilidad están presentes el sujeto que interacciona desarrollando la habilidad, el objeto o sujeto sobre el que interactúa el primero, el objetivo con que actúa y un sistema de operaciones.

Otros plantean como componente de la habilidad a su imagen generalizada o base orientadora de la acción, considerando que la habilidad se debe asimilar a partir de una imagen de las acciones a realizar dada de antemano (Talízina, 1984); lo cual en opinión de los autores, es contraproducente, ya que si la habilidad debe ser erigida en el propio proceso de enseñanza aprendizaje, generalizando el estudiante la misma en su actividad e interacción con el grupo, la imagen generalizada se construye conjuntamente con la habilidad durante el proceso y no como parte de la estructura de la habilidad en sí.

Se puede concretar que la estructura de la habilidad consta de:

- Sujeto (el que realiza la acción).

- Objeto (el que recibe la acción del sujeto).

- Objetivo (aspiración consciente del sujeto) - sistema de operaciones (estructura técnica de la habilidad)

La habilidad se identifica, en el plano psicológico, con las acciones que deben ser dominadas en el proceso de aprendizaje, las que al ser llevadas al proceso de enseñanza - aprendizaje, se convierten en contenido del mismo.

Las acciones que realiza el hombre cuando éste tiene un dominio de las mismas, constituyen una habilidad. Esta acción modelada se convierte en el contenido que debe ser asimilado por el sujeto en su proceso de aprendizaje; ahora se comprende de manera clara la relación entre habilidad y acción.

Se requiere, consideran los autores de la modulación de las acciones y sistematización del proceso, lo que implica, una selección y estructuración de las habilidades, así como el proceso de sistematización de 
las mismas (asimilación y profundidad) por parte de los estudiantes.

Analizando los criterios de Talízina (1984) al caracterizar la habilidad atendiendo a sus elementos: además del conjunto de operaciones que la integran y constituyen su estructura técnica, hay que tener en cuenta al sujeto que debe dominar la habilidad, el objetivo que se satisface mediante la habilidad, la orientación que determina la estructura de dicha acción y el resultado que se ha de esperar de la acción (que tiene que coincidir con el objetivo).

Lo que permite a los autores analizar la clasificación de las habilidades dentro de la estructura como sistema dentro del proceso enseñanza-aprendizaje.

\section{Clasificación de las habilidades}

Dentro de la estructura del sistema de habilidades, se precisa una clasificación del sistema de habilidades, a partir de clasificaciones dadas por Talízina (1984) y Álvarez (1996), según Coll, 2008), las habilidades se deben clasificar en tres grupos a saber:

- Habilidades específicas, propias de las ciencias, de las profesiones o de las tecnologías que son objeto de estudio o trabajo.

- Habilidades lógicas o intelectuales.

- Habilidades de comunicación.

Existen otras clasificaciones, pero en todas hay como rasgo común, el que las habilidades son el resultado de un proceso de aprendizaje, que tienen que transitar por determinados niveles de la sistematización.

Sí consideramos un proceso de formación inductivo y no deductivo obtendremos como resultado el desarrollo de las capacidades de los estudiantes.

El primer nivel de habilidad, en una ciencia o tecnología dada, lo identificamos como la habilidad elemental; ésta se sustenta en conocimientos de esa ciencia, tecnología o arte y en habilidades primarias, que actúan como operaciones dentro de esta habilidad elemental.

Dicho en otras palabras, si una habilidad elemental se descompone en operaciones, pero estas operaciones no serán de la propia ciencia, tecnología o arte, sino que pertenecen a otras, constituyendo habilidades primarias respecto a las elementales.

Siempre la habilidad primaria, tendrá un carácter relativo respecto a las habilidades elementales que son específicas, esto quiere decir que, una determinada habilidad puede comportarse en determinado caso como habilidad primaria, o como habilidad específica, dependiendo de la rama del saber que se considere. Constituyen habilidades primarias, las habilidades lógicas, motrices, o de otras ramas del saber.

Sólo las habilidades lógicas, están siempre constituidas por operaciones que también son lógicas. Esto se fundamenta en trabajos realizados por investigadores, en que se justifica el carácter inseparable de las habilidades lógicas en el proceso del pensamiento, donde se dan de manera integrada, existiendo una mutua dependencia entre ellas.

Hay una interrelación entre el conocimiento y la habilidad; en particular, en las habilidades lógicas se requieren consideraciones especiales.

El vínculo entre habilidad y conocimiento, no queda en la habilidad primaria y la habilidad elemental; en la medida en que se van sistematizando las habilidades también se sistematizan los conocimientos. En el propio proceso se dan ambas, sobre la base de la sistematización de las habilidades, se logrará la de los conocimientos.

Las habilidades lógicas (del pensamiento), no se corresponden de manera directa, con una determinada disciplina o ciencia concreta, sino que cada disciplina puede y debe contribuir a su formación. Claro está, cada disciplina formará aquellas habilidades lógicas que se avienen a las características del objeto de estudio de la misma.

Las habilidades lógicas son múltiples, sólo las más usadas constituirían un gran listado, pero es posible a partir de la formación intencional de algunas de ellas, asegurar la formación del pensamiento lógico, pues estas habilidades no se dan de forma aislada, sino muy relacionadas entre sí durante el proceso de su apropiación y aplicación a los contenidos de las disciplinas.

En el proceso de apropiación del contenido, están presentes el análisis, la síntesis, la comparación, la abstracción, etc. entrelazadas mutuamente. Para algunos "el pensamiento se dirige para resolver los problemas a los procesos mentales vinculados entre sí y que se funden uno en otro." (J. Rubinstein, ).

La relación conocimiento-habilidad específica y de pensamiento lógico, se va perfeccionando en dos direcciones en el proceso de enseñanza-aprendizaje, construyendo habilidades de mayor nivel de sistematicidad. Las cuales son:

I. A través de un proceso consciente que permite cumplir acciones teóricas y prácticas de mayor complejidad, lo cual se produce en el enfrentamiento de mayor riqueza.

II. A partir de un proceso de ejercitación, donde enfrentan problemas de igual grado de complejidad, de manera que se automaticen las 
habilidades, siendo el sujeto cada vez menos consciente de sus acciones, formando un hábito.

Esto permitirá abordar la formación de algunas habilidades dentro de la carrera de Derecho, a partir del plano teórico up supra.

\section{La formación de algunas habilidades en el estudiante de la carrera de Derecho en la Universidad Cubana}

Esta situación a la que se enfrenta la población mundial a principios del milenio, se traduce en un desafío para los educadores de todo el mundo, como los encargados de formar a las nuevas generaciones para cumplir su encargo social en este siglo XXI.

Es por ello, que Cuba enfrenta nuevos y mayores retos, entre ellos la de formar ciudadanos capaces de procesar el caudal de información actual, comprender y actuar conscientemente en la solución de los problemas.

Esto requiere un alto grado de habilidades como la observación, la obtención de información, la planificación de la misma, la solución de problemas y la comunicación de sus resultados, entre otras, que como procedimientos generales, le permitan profundizar en el conocimiento de la realidad y determinar sus características, establecer sus nexos, sus regularidades, es decir, poder conocer y fundamentar lo válido o no de sus productos.

Ello exige una Universidad y un profesor que apliquen, en el proceso de enseñanza-aprendizaje en la formación del estudiante de Derecho, los métodos y procedimientos de la investigación, la dinámica de la ciencia y sobre todo, estos exigen mayor sistematización e integración de los resultados de la investigación, los que se apropian con las habilidades en el quehacer del estudiante que su resultado sea un profesional con competencias.

Por ello una de las tareas primordiales de los primeros años de la Revolución fue la realización de una verdadera Reforma Universitaria. Esta planteó como uno de sus principios la vinculación de la enseñanza y las investigaciones científicas y desde 1959 Fidel Castro Ruz, impulso la idea de la universalización del conocimiento, entendida como la oportunidad de todos los cubanos de estudiar durante toda la vida.

Para lograr este objetivo se adoptaron un conjunto de medidas entre ellas, la Campaña Nacional de Alfabetización, se convirtieron los cuarteles en escuela, se implantó el Sistema de Educación para adultos con la apertura de Secundarias y Facultades Obreras Campesinas y con relación a la Enseñanza Superior se brindaron cursos de nivelación para facilitar el ingreso a las universidades en toda la nación, además se crearon cursos universitarios ofrecidos en horarios especialmente diseñados para los trabajadores. Todas estas medidas en conjunto hicieron realidad el derecho del pueblo a la Educación regulado desde nuestro texto constitucional patrio.

La década el 70 del siglo pasado fue también importante para el desarrollo de la universalización de la Universidad, en término de expansión y ampliación de la red de instituciones (las Pedagógicas, Ciencias Médicas, Universidad de Artes, de Educación Física y Deporte, etc.) que se tradujo en una ampliación de la matrícula universitaria, de programas de estudios de posgraduados y del inicio de la modalidad de Educación a Distancia, y se creó con ello el Ministerio de Educación Superior dentro de los organismos de la Administración Pública.

El pasado siglo XX mostró una modificación considerable en las características de la actividad científico investigativa en la enseñanza y esto propició que el significado de las orientaciones con apoyo en los métodos de la ciencia constituyera una prioridad en la forma de enseñar las ciencias jurídicas, además uno de los rasgos distintivos fundamentales de la investigación científica, tecnológica y humanística en la Educación Superior Cubana, por su vinculación estrecha con las prioridades del desarrollo socioeconómico nacional, que permitieron con ello buscar respuestas a las necesidades socioeconómicas.

En el siglo XXI, se muestran nuevos retos para la enseñanza superior en la nación, uno de estos en la transformación del proceso de enseñanza aprendizaje en la carrera de Derecho.

Ya expuesta las notas características de las habilidades, vistas desde un plano teórico, es saludable analizar como alcanzan éstas su vitalidad durante el transcurso de el proceso de enseñanza-aprendizaje de la carrera Derecho han ido en evolución. Tomado a partir de:

Habilidades Específicas: (ligadas a una rama de la cultura o profesión): son el tipo de habilidad que el sujeto desarrolla en su interacción con el objeto de estudio o trabajo concreto y que en el proceso de Enseñanza-Aprendizaje, una vez que son suficientemente sistematizadas y generalizadas, se concretan en métodos propios de los diferentes objetos de la cultura que se configuran como contenido.

Lo anterior es de vital importancia en el proceso de enseñanza del derecho, pues si bien al inicio del presente este trabajo, afirmamos, que se debe formar más que informar, es dable preguntarse qué debemos formar.

La respuesta surge por sí sola: Juristas antes que prácticos; dotados de conocimientos que los conviertan 
en científicos antes que técnicos, que sean capaces de comprender y adaptar las normas que rigen la realidad social de la comunidad a cada transformación que sufra ésta, pues es únicamente el jurista quien estará preparado para adecuar las normas a los nuevos requerimientos. Tomando como punto de análisis los conocimientos adquiridos con teoría general del Derecho.

Al jurista que se forma en la Universidad patria se le deben crear habilidades, que logren ver al derecho desde el punto de vista normativo y desde el punto de vista de la realidad jurídica, ya que estos se implican recíprocamente, pues la norma escrita, como principio básico general de la legalidad, solo determina apriorísticamente, las formas del comportamiento humano que estarán prohibido o permitido posterior a su promulgación, por lo que cada uno de sus operadores tienen como misión saber como se adecua cada comportamiento humano a estos principios generales.

Desde que el estudiante de Derecho recibe sus primeras clases, debe el profesor potenciar habilidades, entiéndase, determinadas por la relación sujeto-objeto, en función de los problemas que debe resolver, durante su transito por los distintos años de la carrera, así como una vez graduado, las cuales estarán asociadas al tipo de conocimiento:

Para el caso de un conocimiento efectivo, las habilidades asociadas a éste son:

- Caracterizar los rasgos fundamentales que se observan.

- Precisar las condiciones en que ocurre.

- Establecer su esencia y el mecanismo de su desarrollo.

- Utilizar en ejemplos prácticos.

Para el caso de conceptos:

- Denominar

- Definir

- Interpretar

- Comparar

- Clasificar

Para el caso de conocimientos relacionales tales como leyes o principio:

- Precisar y explicar rasgos que caracterizan la ley.

- Establecer e interpretar las relaciones cualitativas entre las características que intervienen.

- Analizar los límites de aplicación de la ley.

- Aplicar la ley en la explicación de fenómenos conocidos o nuevos.

No obstante, se justiprecia que las disciplinas y asignaturas básicas y básicas específicas, también ocupan un lugar importante en este proceso, por cuanto las habilidades que aportan, aun cuando no se identifiquen con las habilidades profesionales y modos de actuar del profesional, pueden corresponderse con éstos, al aportar -como en el caso de la Teoría del Derecho - una lógica de actuación profesional.

Habilidades Lógicas: son las que permiten asimilar, comprender y construir el conocimiento, guardan una estrecha relación con los procesos fundamentales del pensamiento como son análisis, síntesis, abstracción, concreción y generalización. Se desarrollan a través de habilidades específicas. Están en la base del desarrollo del resto de las habilidades y en general de toda actividad cognoscitiva del hombre.

El jurista se forma a partir de la preparación profesional y la investigación, eso requiere de basamento teórico y ejercitación práctica. Es incuestionable que la prioridad estará dada por el estudio de la legislación y jurisprudencia vigentes en un país determinado y su proyección práctica.

Pero no es menos cierto, valoran los autores que serán ineludible el conocimiento de la Teoría general del Derecho, su vinculación interdisciplinaria y su basamento filosófico.

En lo anterior se aprecia cómo se dejó sentado que una de las vías de formar al jurista es a través de su ejercitación práctica, lo cual constituye en consecuencia un desafío, para configurar ésta, esta ha recibido el nombre de sistema de enseñanza de la práctica forense o clínica jurídica, juegos de rol, siendo este último la variante que se implementa en el la realidad cubana, como parte del ejercicio profesional, con un amplio sentido de integridad de la ciencia del Derecho Procesal, su técnica, la ética en el ejercicio y su vinculación entre sí y la conexidad con otras ciencias, sistemas y tecnologías.

Para ello se ha trabajado o determinado cuatro niveles de la materia ejercicio profesional en la carrera de Derecho, que son:

El primero de ellos se ubica en el primer año de la carrera, designada la materia como "Ejercicio Profesional I "generando un aprendizaje orientado a familiarizar al alumno con los distintos organismos básicos, donde ser puedan ellos desarrollar como operadores jurídicos, (Fiscalía General de la Republica, Tribunales, Bufetes Colectivos, fundamentalmente), haciendo especial énfasis en la actividad que allí se realiza y el papel que juega este dentro de la sociedad, sus normativas de organización, así como la regulación de la ética profesional de los que allí laboran.

El segundo nivel en el segundo año de la carrera que 
responde a la denominación de "Ejercicio Profesional II", que tiene como objetivo, evaluar la conformación, organización y funcionamiento de los órganos del Estado en particular los de carácter representativos y fiscales, destacando aciertos e insuficiencias con vistas a la solución de los problemas detectados.

Valorar, sobre la base de los conocimientos adquiridos y a partir de su constatación empírica, la participación popular en la conformación y funcionamiento del poder estatal. Dominar la tramitación y solución de las quejas de la población por lesiones a los derechos ciudadanos en las fiscalías municipales y provinciales como mecanismo de protección de los derechos de los ciudadanos y de la supremacía del texto constitucional que los contiene. Lo anterior dota al estudiante de un conjunto de habilidades que van desde: Destacar los aciertos e insuficiencias en la actuación de las comisiones y colegios electorales, órganos representativos y fiscales. Proponer solución a los problemas detectados y coadyuvar a la solución de los mismos; observar y participar en la organización y desarrollo del proceso electoral; atender al público en las fiscalías y en los despachos de los delegados de circunscripción; recepcionar, clasificar, tramitar las quejas de la población en las fiscalías; realizar investigaciones socio-jurídicas en las unidades docentes y en las circunscripciones electorales.

El tercer nivel en el tercer año de la carrera que responde a la denominación de "Ejercicio Profesional III", presenta la particularidad de recrear y apoyar de manera interrelacionada lo dictado por la materia Derecho Procesal Civil, generando un aprendizaje orientado al desarrollo e implementación de los procesos de conocimiento, los de ejecución, los especiales, los voluntarios y la resolución de conflictos, a partir del procedimiento de administrativo.

Lo anterior crea en el estudiante habilidades necesarias para saber desempeñarse adecuadamente en los campos de acción del Abogado, del Juez y del Asesor de las Empresas, determinado el tratamiento legal a seguir en cada caso, mediante la integración y aplicación de los conocimientos adquiridos en materia de Derecho Civil y familia y de Derecho Administrativo, sobre una base de los fundamentos y regulaciones de la ética profesional.

El cuarto y último nivel en el cuarto año de la carrera que responde a la denominación de "Ejercicio Jurídico IV" se encuentra dividido en dos cursos individuales y simultáneos que se interrelacionan y apoyan el dictado de las materias Derecho Procesal Penal y Derecho de Empresa, generando un aprendizaje orientado al desarrollo e implementación de los procesos penales con sus distintas características. Esto mismo se reproduce en el curso con orientación laboral, donde además se recrean los procedimientos extrajudiciales, las actuaciones ante sala de lo económico.

Los estudiantes se apropiarán de habilidades propias de la esfera de actuación donde se encuentren ubicados en su ejercicio profesional (Fiscalía, Tribunales, Bufetes Colectivos y Empresas (sujetos de gestión económica), de igual forma se realizaran juegos de roles, simulación de actos de juicio oral, tomando como base causas ya resueltas, encuentros técnicos y otras actividades complementarias que propiciaran el intercambio de experiencias y permitirán complementar el proceso de habilidades de los educandos.

$\mathrm{Si}$ logramos la habilidades propuestas en los estudiantes, con estos ejercicios jurídicos, rescatamos aquí una visión clásica de la doctrina procesal que se desenvuelve en tres planos: filosófico dirigido a entender el valor justicia, técnico estudiando la norma jurídica en sus menores detalles al servicio de la solución concreta y otra política que aspira a comprender lo que el poder judicial significa en el marco de los poderes del Estado.

Pero ello en función de capacitar a aquellos que en el futuro, a través del ejercicio de su profesión, tienen la responsabilidad de recorrer caminos tendientes a lograr la afirmación de la idea de justicia en la sociedad o que desde los tribunales han de transitarlos para administrarla. Con ello se apunta a afirmar la relación existente entre Derecho y Justicia, al ser esta última por su propia naturaleza un principio de orden. En estos ejercicios jurídicos el estudiante debe aplicar minuciosamente lo aprendido, desde sus inicios en los años de la carrera de Derecho, respecto a la solución de los casos, y que ya enunciábamos cunado explicábamos lo referente habilidad específica, consiste, en la necesidad que tiene el estudiante como interprete de acudir al sustrato de lo normativo, para lo cual se valdrá de la doctrina, leyes o códigos, lo cual solo lo dotará de un conocimiento superficial y aislado, debiendo estar seguido de las explicaciones del profesor o tutor y la nueva lectura de la norma, para de esta forma, ondear más en el significado; posteriormente debe ir al caso concreto y analizar sus particularidades.

No siendo lo anterior suficiente, pues el conocimiento práctico del hombre se da a través de un silogismo, a partir del cual la norma como principio general y el hecho concreto se unen nuevamente, lo que demuestra, que el aprendizaje práctico de los principios generales (premisa mayor) sólo se alcanza a través de casos determinados. Siendo necesario estudiar separadamente y luego recíprocamente hasta que se logre realizar una interpretación correcta de la norma para una adecuada aplicación al caso concreto.

Habilidades del procesamiento de la información y comunicación: son las que permiten procesar la 
información, incluyéndose aquellas que permiten obtener la información y reelaborar la información. Aquí se incluyen aquellas habilidades del proceso docente como: tomar notas, hacer resúmenes, así como exponer los conocimientos tanto de forma escrita como oral, de suma importancia ella, para que el estudiante alcance independencia cognoscitiva. En el contexto de la Didáctica de la Educación Superior en la nación cubana, se precisa de un tipo específico de habilidad, que se forma en dicho contexto y que forman la base de la actuación profesional, en este caso se trata de las habilidades profesionales, que constituyen el contenido de aquellas acciones del sujeto orientadas a las transformaciones del objeto de la profesión.

Este el tipo de habilidad que deberá sistematizarse hasta generalizarse con un grado tal que le permita aplicar los conocimientos, actuar y transformar su objeto de trabajo y resolver los problemas más generales y frecuentes que se presenten en las diferentes esferas de actuación. Constituyen por consiguiente la esencia de la actuación del profesional y punto de partida del modelo del profesional.

La apropiación de habilidades generales, conduce a la formación de un pensamiento teórico, es decir, que se pueda operar con generalizaciones teóricas, con conceptos, leyes, principios generales, con la esencia del conocimiento. Si solo se desarrollan habilidades específicas, el tipo de pensamiento que se forma es empírico.

Se plantea que algunas de las habilidades generales que la didáctica actual debe contribuir a desarrollar mediante procedimientos adecuados sean:

Habilidades relacionadas con acciones intelectuales: observación, descripción, determinación de las cualidades (generales, particulares y esenciales), comparación, clasificación, definición, explicación, ejemplificación, argumentación, valoración, solución de problemas, modelación, elaboración de preguntas, planteamiento de hipótesis, entre otras.

El estudiante deberá poner en función de la investigación jurídica los conocimientos adquiridos en las diferentes asignaturas de la carrera, lo cual crea una habilidad de investigación aplicada.; al constituir por si misma una continua generación de hipótesis de investigación, las que obligan a profesores y estudiantes a emplear todas las herramientas a su alcance en pos de una adecuada transformación o actualización de la normativa de diferentes instituciones jurídicas.

Lo anterior señalado, muestra algunas de las destrezas que se pueden fundar en los estudiante, las cuales para su mejor exposición y comprensión fueron separadas, pero algunas de ellas se pueden constituir al unísono en los educandos, a través de procesos inductivos, las cuales estarán determinadas en el orden académico según las materias y ejercicios jurídicos que reciban, para al final lograr formar un estudiante con habilidades formadas, no habiendo quedado éstas, en el plano de la mera información.

Uno de los elementos fundamentales del sistema de trabajo en la Educación Superior, es la actividad científica estudiantil. La importancia de este problema crece ante las exigencias que imponen la época y la necesidad de contar con profesionales capaces de impulsar el desarrollo científico del país.

Lo que se evidencia con los resultados en las jornadas científicas estudiantiles en los estudiantes de la carrera de Derecho, demuestran la necesidad de trabajar por el desarrollo de habilidades investigativas, pues no hay dudas que para alcanzar la pertinencia exigida por la universidad resulta imprescindible la formación de cualquier profesional como investigador, ésta necesidad se manifiesta especialmente en la carrera de Derecho; es imprescindible que los egresados sean capaces no solo de aplicar la norma del Derecho positivo, sino de mejorarlo y adecuarlo a las nuevas situaciones, que puedan contribuir al perfeccionamiento tanto de nuestro sistema jurídico como a la creación del nuevo Derecho y para lograr estos resultados, el profesor universitario tiene la misión de prepararlo para ello.

Este primer esbozo permitirá refrendar que la investigación en Cuba ha sido uno de los temas tratados con sistematicidad en el pensamiento educativo desde el siglo XIX hasta la actualidad en el siglo XXI. Resulta claramente comprensible que las primeras manifestaciones de lo antes expuesto se hallen en la petición de reformas a España para tener en Cuba una educación científica, práctica y vinculada con las necesidades propias.

Resaltan como evidencia de lo señalado entre los más destacados educadores cubanos del siglo XIX que expresaron su preocupación y oposición a la vieja enseñanza memorista y al adiestramiento autoritario y se pronunciaron en defensa de un aprendizaje activo por parte de los estudiantes académicos de la talla de José Agustín Caballero, Félix Valera, José Martí y Enrique José Varona, como referentes en la educación cubana.

Es así, que en el pensamiento del más universal de los cubanos, José Martí observara en que su concepto de educación es abarcador y amplio, múltiple y totalizador. Al igual que sus antecesores, Martí abogaba por una preparación eficaz del hombre para la vida en la época que le correspondía y por ello mencionaba como un crimen el divorcio entre la educación que se brindaba y la época en que se vive. 
Martí comprendió la necesidad de dar un cambio radical a la universidad para que la misma cumpliera sus funciones sociales, por ello planteaba la necesidad de que se hiciera corresponder al mundo nuevo la universidad nueva, ya que la universidad no podía quedar a la zaga del desarrollo que experimentaban las ciencias.

Es criterio de los autores, que es este uno de los paradigmas que hoy tiene como reto la nueva Universidad cubana en el siglo XXI, el graduar estudiantes con competencias para el mercado laboral cubano en la actualización del modelo económico, del que no están exentos los profesionales del Derecho.

\section{CONCLUSIONES}

Que el problema del dominio de las habilidades y de los conocimientos asociados a éstas supone la responsabilidad de concebir y ejecutar estratégicamente un proceso docente que en su dinámica garantice la apropiación eficiente de las mismas, al estimular y potenciar el desarrollo individual e integral del estudiante de la carrera de Derecho.

Que las habilidades siempre serán, resultado del aprendizaje y éstas se desarrollan en el proceso de interacción del estudiante de Derecho (sujeto) con la sociedad, a partir del silogismo que se produce, de la confrontación de la teoría y la práctica.

Que la articulación de un programa en la carrera de Derecho debe estar orientado a formar habilidades, que le propicien al estudiante las herramientas, los conocimientos y la cultura adecuada para el futuro ejercicio como profesional, todo ello desde el ámbito universitario para hacerlo más competente en las habilidades básicas en el mercado laboral en el siglo $\mathrm{XXI}$ donde se pondera el profesional competente.

La educación en Cuba como sistema, debe a futuro ser regulada en una norma jurídica con rango de Ley cumplimentándose el desarrollo normativo del país en la materia educativa.

\section{REFERENCIAS BIBLIOGRÁFICAS}

Álvarez, D. C. (1996). La escuela en la vida. Colección Educación y Desarrollo y del mismo autor. La Habana: Pueblo y Educación.

Álvarez, I. (1995). Perfeccionamiento de la formación de habilidades en la solución de problemas de Física para estudiantes de Ciencias Técnicas. Tesis de Maestría en Ciencias de la Educación.

Álvarez, I. (1999). El proceso y sus movimientos: Modelo de la Dinámica del Proceso Docente Educativo en la Educación superior. Tesis en opción al grado científico de doctor en Ciencias Pedagógicas, CEES "Manuel F. Gran" Universidad de Oriente.

Aneca. (16 de marzo de 2006). Libro Blanco del Título de Grado en Derecho. Obtenido de http://www. aneca.es/activin/docs/libroblanco_derecho_def. pdf

Bretel, L. ( 16 de abril de 2010). Evaluación de Competencias. Obtenido de :http:// breteleandocompeten.blogspot.com/2005/09/ evaluacin-de-competencias.html

Coll Aguilera, M. (2008). Estrategia didáctica para la sistematización de las habilidades generales más aplicadas en la disciplina principal integradora de la carrera de derecho. Tesina en opción al título académico de especialista en docencia universitari, Universidad de Granma, Cuba.

Derecho, F. d. (2010). Plan de estudio de la carrera de Derecho D perfeccionado. Cuba: MES.

Díaz, B. (1992). Didáctica: Aportes para una Polémica. Aique, Buenos Aires, Argentina.

Fuentes , H., \& Cruz, S. (1997). Curso Diseño Curricular. Monografía, CEES Manuel F. Gran, Santiago de Cuba.

Fuentes, H., Álvarez , I., \& Cruz, S. (1998). Modelo holístico configuracional de la Didáctica de la Educación Superior. Monografía, CEES "Manuel F. Gran" - Universidad de Oriente.

Fuentes, H. (1998). Modelo holístico configuracional, como una aproximación a la Didáctica. . CEES "M. F. Gran".

Fuentes, H., Pérez, L., Mestre, U., \& otros, y. (1994). La formación de habilidades a través de la solución de problemas . Monografía, "Manuel F. Gran". ISPJAM.

González Losada, S. (2010). Análisis de las competencias de los estudiantes de Derecho en los nuevos planes de estudio de Andalucía en el EEES. Revista de Educación(12).

Nisbet, J., \& Schucksmiths. (1987). Estrategias de aprendizaje.

Novak, J. D., \& D, B. (1984). Aprendiendo a aprender - Barcelona: Original Learninghow to learn. Cambridge, Ma: Cambridge University Press.

Real Decreto 1393/2007 por el que se establece la ordenación de las Enseñanzas Universitarias Oficiales. BOE, no 260, . (30 de Octubre de 2007).

Witker, J. (s.f.). e: Antología de estudios sobre la enseñanza del derecho. Obtenido de www. juridicas.unam. $\mathrm{mx} / \mathrm{inst} / \mathrm{direc} /$ datper.htm- $\mathrm{p}=$ witker. $\mathrm{htm}$

Zilberstein , T. J., Herrero , T. E., Borroto, C. G., \& y otros . (2006). Preparación pedagógica integral para profesores integrales. Félix Varela. 